\title{
ESTADO ATUAL DA LEISHMANIOSE CUTÂNEA DIFUSA (LCD) NO ESTADO DO MARANHÃO. II. ASPECTOS EPIDEMIOLÓGICOS, CLÍNICO-EVOLUTIVOS
}

\author{
Jackson Mauricio Lopes Costa, Ana Cristina Rodrigues Saldanha, Ana Carla \\ de Melo e Silva, Artur Serra Neto, Clovis Eduardo Santos Galvão, Conceição \\ de Maria Pedroso e Silva e Antonio Rafael da Silva
}

\begin{abstract}
Os Autores fazem um estudo retrospectivo e prospectivo de 6 pacientes portadores de leishmaniose cutânea difusa, observados no Estado do Maranhão a partir de 1974. Os casos abordados são oriundos de diversas regiôes do estado, observando-se em todos eles o envolvimento da leishmania (Leishmania) amazonensis, sendo que 5 (84\%) dos pacientes apresentaram início de doença na $l^{a}$ década de vida. Em todos os pacientes envolvidos no estudo, houve relato de lesão inicial nodular única, que, posteriormente, em periodo variável de tempo, disseminou-se adquirindo outros aspectos. Evolutivamente apresentaram múltiplas lesões nodulares e ulceradas, intradermorreação de Montenegro(-) e refratariedude aos esquemas terapêuticos utilizados até ao presente momento.
\end{abstract}

Palavras-chaves: Leishmaniose cutânea difusa. Leishmania (Leishmania) amazonesis. Estado do Maranhão.

As primeiras descrições da leishmaniose cutânea difusa (LCD), sob o ponto de vista clínico, histopatológico e parasitológico, ocorreram no Brasil em $1945^{32}$ e na Venezuela no ano de $1946^{12}$, posteriormente, Barrientos ${ }^{3}$ na Bolívia, descreve um caso da doença, chamando atenção para a evolução crônica e a refratariedade em relação à terapêtutica utilizada naquele período. Esta variante da leishmaniose tegumentar americana aparece esporadicamente em áreas onde a doença é endêmica. No Brasil, somente foram observados pacientes nas regiões Norte e Nordeste, destacando os Estados do Amazonas, Pará, Maranhão, Pernambuco e Bahia 1527282931 .

A LCD, foi descrita também na Venezuela, Bolívia, República Dominicana, México, Colômbia, Honduras, Costa Rica e Estados Unidos, sendo que fora das Américas somente a Etiópia destacou-se com 33 casos 36791416 .

Das 10 espécies de Leishmânias que causam doença em humanos, o complexo mexicana encontra-se estritamente associado à leishmaniose

\footnotetext{
Núcleo de Patologia Tropical, Departamento de Patologia da Universidade Federal do Maranhāo, São Luis, MA. Endereço para correspondência: Prof. Jackson Maurício Lopes Costa. Núcleo de Patologia Tropical/FM/UFMA. Pavilhão Pedagógico, Pça. Madre Deus, 02 - Anexo ao Hospital Geral Tarquínio Lopes Filho) 65025-560 São Luís MA.

Recebido para publicação em 20/01/92.
}

cutânea difusa no Continente Americano ( $L$. amazonensis, L. mexicana, L. pifanoi são espécies identifcadas); já na África, temos $L$. tropica e $L$. aethiopica, responsabilizadas pela doença naquele continente 67181920

Na Amazônia brasileira o ciclo ecológico da LCD encontra-se bem estudado. Segundo Lainson \& Shaw, o reservatório primário da Leishmania (L.) amazonensis é o Proechimys guyanensis, tendo como secundários os marsupiais e outros roedores. O vetor é o Lutzomyia flaviscutellata, flebotomíneo de hábitos noturnos, de vôo baixo que geralmente não se atrai pelo homem, que é um hospedeiro acidental. Portanto, a infeç̧̧̃a humana não é comum, sendo geralmente adquirida pelos indivíduos que penetram nas várzeas, onde a população do vetor é particularmente elevada 1830 .

No que diz respeito aos aspectos clínicos, observa-se que no local da inoculação, forma-se uma mácula, pápula ou nódulo que, ao contrário do que geralmente ocorre na leishmaniose cutânea, não ulcera; depois, aparecem outras lesões semelhantes na vizinhaça e, num período de tempo que varia de alguns meses a anos, há disseminação hematogênica do parasita com aparecimento de lesões em várias áreas do tegumento 10111320212224 .

No Maranhão, a LCD foi descrita pela primeira vez na década de 1960 , sendo hoje o estado brasileiro 
Costa JML, Saldanha ACR, Silva ACM, Serra Neto A, Galvão CES, Silva CMP, Silva AR. Estado atual da leishmaniose cutânea difusa (LCD) no Estado do Maranhão. Il. Aspectos epidemiológicos, clínico-evolutivos. Revista da Sociedade Brasileira de Medicina Tropical 25:115-123, abr-jun, 1992.

com o maior número de casos. Sentindoa necessidade de respostas, para diversas interrogaçōes sobre a evolução da doença no estado, decidimos realizar o presente trabalho, na tentativa de trazer alguma contribuição para o conhecimento desta patologia em nosso meio, dando continuidade ao estudo iniciado por Costa e cols ${ }^{15}$.

\section{MATERIAL E MÉTODOS}

Para a realização deste trabalho, foi feito um estudo retrospectivo e prospectivo de 6 (seis) casos de LCD, acompanhados a partir da década de 70, nos hospitais da rede pública de São Luís, Maranhão. Para uma melhor avaliação do mesmo, fez-se um levantamento dos prontuários, desde a primeira internação à "última avaliação". Para os autores, última avaliação corresponde à observação final realizada pela equipe em relação aos pacientes. Considerando o mês de junho de 1991 como observação final.

Os dados colhidos dos prontuários dos pacientes, destacavam os aspectos clínicos, como: dados pessoais (idade, sexo, raça, naturalidade, procedência, profissão, tempo de doença), dados referentes às lesões (localização, aspecto, ano do aparecimento da primeira lesão), presença ou não de linfonodos palpávêis e evolução clínica no decorrer da doença correlacionando-a com a terapêutica empregada. Em relação ao laboratório, exames realizados para o diagnóstico e isolamento do parasito, intradermorreação de Montenegro, histopatologia, inoculação em hamster (Mesocricetus auratus), esfregaço e cultura em meios artificiais (LIT/NNN) ${ }^{17}$.

No que diz respeito à terapêutica, levamos em consideração a análise dos tratamentos utilizados e a resposta clínica referente a cada esquema. Deu-se ênfase à dose total do $\mathrm{Sb}^{v}$ (substância ativa do antimoniato- $\mathrm{N}$-metilglucamina), e outras drogas opcionais utilizadas no tratamento.

\section{RESULTADOS}

Dos 6 pacientes envolvidos no estudo, $5(84 \%)$ foram acometidos na $I^{a}$ década de vida e apenas 1 $(16 \%)$ apresentou início da doença na $3^{\mathrm{a}}$ década. Observou-se predominância do sexo masculino, 4
(67\%) e, devido ao pequeno número de casos não pudemos considerar relevante a procedência e a naturalidade dos pacientes. O acometimento precoce, notado na maioria dos casos, $5(84 \%)$, não nos permitiu fazer uma relação entre incidência e atividade profissional.

Pudemos constatar o caráter evolutivo crônico da doença nos casos estudados, sendo que em relação aos aspectos clínicos, todos os pacientes apresentaram polimorfismo lesional, com disseminação das lesões; $3(50 \%)$ pacientes apresentaram infiltração na mucosa nasal. Detalhes referentes a estes dados são mostrados na Tabela 1 e Figuras 1, 2, 3 e 4.

Em relação aos exames laboratoriais, intradermorreação de Montenegro negativa, persisitindo atéà última realização da mesma (junho/ 91). Nos6 (100\%), pacientes isolou-se a Leishmania (Leishmania) amazonensis como agente etiológico da doença (Isolamento realizado no Instituto Oswaldo Cruz, Rio de Janeiro, RJ, Grimaldi Jr). Os Dados referentes aos exames laboratoriais encontram-se na Tabela 2.

Quanto à terapêutica, demos ênfase à utilização do antimoniato-N-metilglucamina (glucantime). A dose comprovada do $\mathrm{Sb}^{\mathrm{v}}(\mathrm{g})$ foi obtida a partir dos dados retirados dos prontuários. A correlação entre terapêutica (glucantime), seguimento e resultados clínicos observados na última avaliação encontra-se na Tabela 2. Observou-se que em todos os casos houve um período considerável de tempo entre o aparecimento da primeira lesão e o início do tratamento (Tabela 3).

Todos os pacientes fizeram uso do glucantime na dose variável de 16 a $20 \mathrm{mg} / \mathrm{Sb} / \mathrm{kg} /$ dia em séries intercaladas por períodos variáveis. Três pacientes fizeram uso de anfotericina B associada ao glucantime no início do tratamento, sem resultados satisfatórios. Por ocasião da última avaliação, 5 (84\%) pacientes encontravam-se fazendo uso de gamma interferon recombinante associado ao glucantime, apresentando discreta melhora no quadro clínico. Um dos pacientes, em determinado período do tratamento, utilizou metronidazol e sulfona (dapsona) sem, no entanto, apresentar melhora no quadro clínico, encontrando-se atualmente no Instituto Evandro Chagas em Belém (PA), fazendo uso da medicação pentamidina, apresentando ainda lesōes clinicamente ativas, entretanto com melhora 
Costa JML, Saldanha ACR, Silva ACM, Serra Neto A, Galvão CES, Silva CMP, Silva AR. Estado atual da leishmaniose cutânea difusa (LCD) no Estado do Maranhão. II. Aspectos epidemiológicos, clínico-evolutivos. Revista da Sociedade Brasileira de Medicina Tropical 25:115-123, abr-jun, 1992.

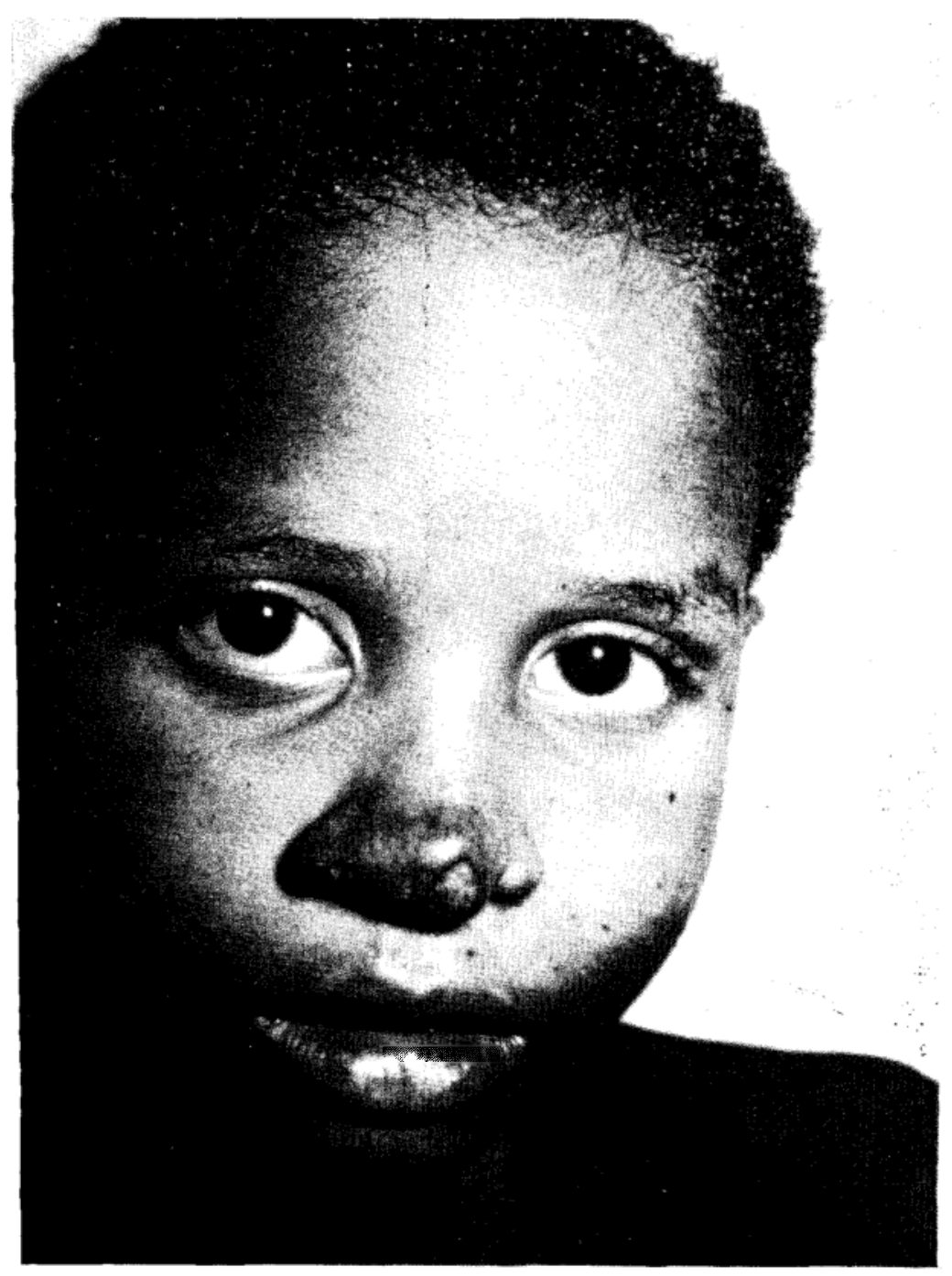

Figura 1 -Aspectos da lesão do paciente A.A.A. aos 6 anos de idade, mostrando detalhes do comprometimento nasal com leve ulceração na asa do nariz (lado esquerdo) associado à infiltração nasal.

do quadro geral (Fernando T. Silveira: informação pessoal).

Durante a evolução clínica, os pacientes apresentaram periodos intercalados de melhora e exacerbação das lesões. Na última avaliação (junhol 91), observou-se presença de lesões clinicamente ativas em todos os casos.

\section{DISCUSSÃo}

Segundo Convit e cols ${ }^{11}{ }^{14}$, os critérios para estabelecer o diagnóstico da LCD são os seguintes: há sempre uma lesão inicial, que evolui, depois aparecem outras lesões semelhantes com posterior disseminação para outras partes do corpo, envolvendo grandes áreas; as lesões são nodulares e não ulceradas; existe abundância parasitária nas lesões; histologicamente caracteriza-se por intenso número de macrófagos parasitados; não há comprometimento visceral; intradermorreação de Montenegro negativa; doença com evolução crônica; a terapêutica com antimonial pentavalente produz somente melhora temporária.

Levando-se em consideração esses critérios, 
Costa JML, Saldanha ACR, Silva ACM, Serra Neto A, Galvão CES, Silva CMP, Silva AR. Estado atual da leishmaniose cutânea difusa (LCD) no Estado do Maranhão. II. Aspectos epidemiológicos, clínico-evolutivos. Revista da Sociedade Brasileira de Medicina Tropical 25:115-123, abr-jun, 1992.

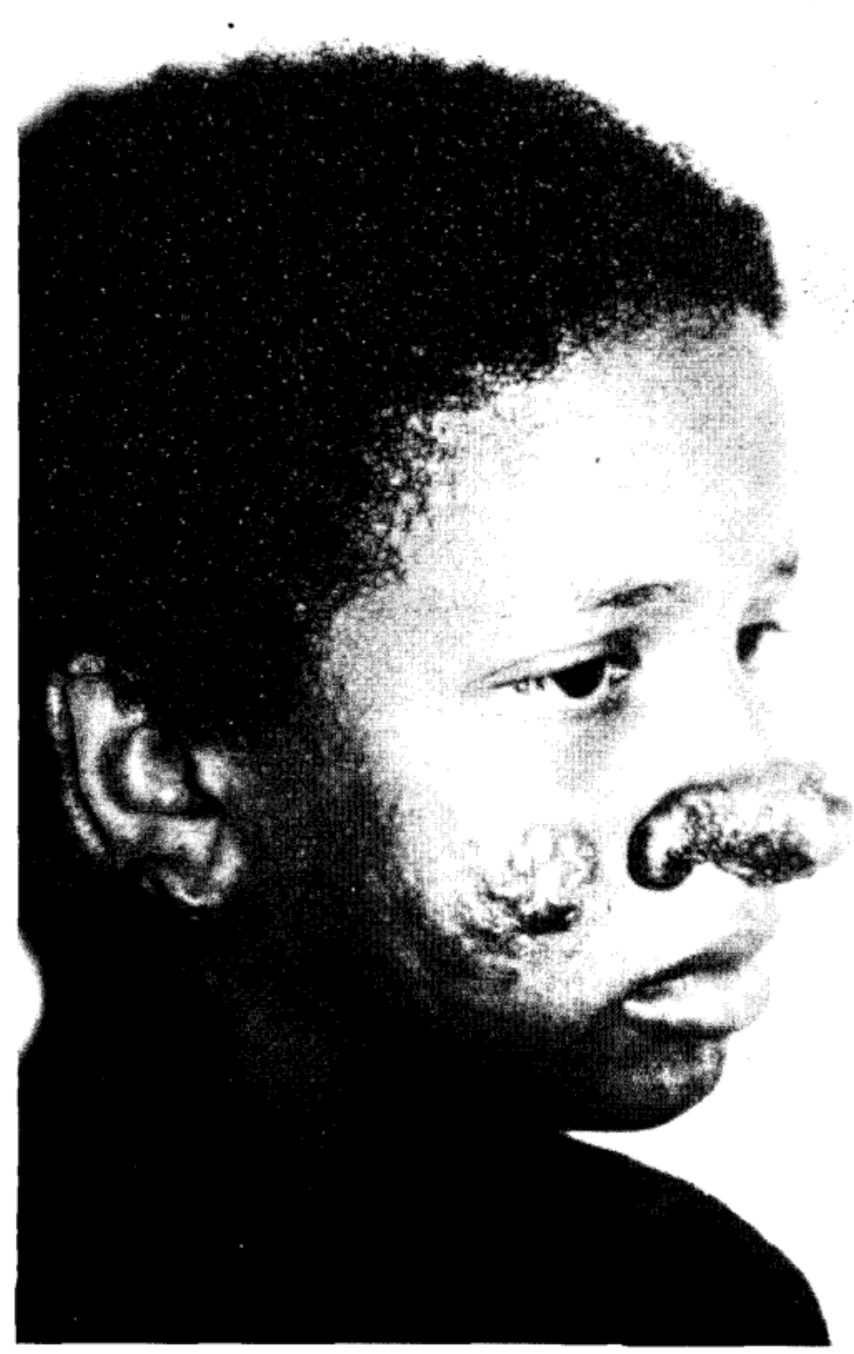

Figura 2 - Paciente A.A.A. aos 13 anos de idade, mostrando detalhes evolutivos da lesão nasal associada à exulceração. Observa-se também comprometimento do pavilhão auricular e seios da face.

faremos uma análise comparativa dos pacientes estudados neste trabalho, na tentativa de estabelecer alguns padrões para LCD no Estado do Maranhão.

As características clínicas dos nossos casos confirmam em parte as descrições clássicas. Todos os pacientes envolvidos no estudo apresentaram lesão inicial localizada nodular que, posteriormente, em espaço variável em tempo, disseminava-se acometendo principalmente face, extremidades superiores e inferiores, não poupando, entretanto, outras áreas, como no paciente (R.N.P.S.) onde houve disseminação em todo o tórax. Quanto ao comprometimento de órgãos profundos, nada constatamos, inclusive em nenhum dos casos houve história pregressa de leishmaniose visceral.

Segundo Bittencout e cols ${ }^{4}{ }^{5}$, quando há ulceração na LCD, sugere sempre causas secundárias como traumas e infecções, devido à elasticidade tecidual facilitar os microtraumas e consequentemente a penetração de microorganismos existentes na pele para o sítio da lesão. O acometimento da mucosa em nossos casos restringiu-se a infiltração e exulcerações da mucosa nasal, embora Barrientos relate "fungosidades"e ulcerações do palato duro, 
Costa JML, Saldanha ACR, Silva ACM, Serra Neto A, Galvâo CES, Silva CMP, Silva AR. Estado atual da leishmaniose cutânea difusa (LCD) no Estado do Maranhão. Il. Aspectos epidemiológicos, clínico-evolutivos. Revista da Sociedade Brasileira de Medicina Tropical 25:115-123, abr-jun, 1992.

Tabela 2 - Detalhes dos aspectos laboratoriais e evolutivos dos pacientes portadores de leishmaniose cutânea difusa estudados.

\begin{tabular}{lllllll}
\hline Pacientes & I.D.R.M. & $\begin{array}{l}\text { Método de } \\
\text { demonstração } \\
\text { do parasita }\end{array}$ & $\begin{array}{l}\text { Caracteri- } \\
\text { zação do } \\
\text { parasita* }\end{array}$ & $\begin{array}{l}\text { Terapêutica } \\
\left(\mathrm{Sb}^{v} / \mathrm{g}\right) \text { total } \\
\text { dose mínima } \\
\text { comprovada }\end{array}$ & $\begin{array}{c}\text { Follow up } \\
\text { (anos) }\end{array}$ & $\begin{array}{l}\text { Resultados } \\
\mathrm{C}=\text { curado } \\
\mathrm{A}=\text { ativo }\end{array}$ \\
\hline
\end{tabular}

$\begin{array}{lcllccc}\text { MECS } & (-) & \text { HI,HA,E } & \text { LLA } & 22 & 13 & \text { A } \\ \text { RNMG } & (-) & \text { HI,HA } & \text { LLA } & 23 & * * & \text { A } \\ \text { AAA } & (-) & \text { HI,HA,E,C } & \text { LLA } & 275 & 8 & \text { A } \\ \text { RNPS } & (-) & \text { HI,E,C } & \text { LLA } & 260 & 2 & \text { A } \\ \text { MCMA } & (-) & \text { HI,E,C } & \text { LLA } & 70 & 1 & \text { A } \\ \text { ROF } & (-) & \text { HI,E,C } & \text { LLA } & 36 & * & \text { A }\end{array}$

Chave de localização: HI - Histopatologia; C - Cultura; E - Esfregaço; HA - Inoculação Hamster; LLA - Leishmania (Leishmania) amazonensis.

* Isolamento e caracterização das cepas (Grimaldi Jr.) (Instituto Oswaldo Cruz, Rio de Janeiro-RJ).

** Pacientes não foram acompanhados por um período variável de 4-7 anos antes de serem encaminhados ao nosso Serviço.

semelhantes às observadas no tipo "espundia"3.

A intradermorreação negativa, constatada em todos os nossos pacientes, atesta a deficiência da imunidade celular específica dos casos, frente aos antígenos parasitários, proporcionando um acúmulo de leishmânias nas lesões e a manutenção de altos níveis de antígenos, provavelmente, responsáveis pela perpetuação da ausência da resposta específica 242526 .

Estudos imunológicos realizados na Etiópia em portadores de LCD demontraram níveis normais de imunoglobulinas, repostas positivas à lepromina $\mathrm{e}$ tuberculina, embora o teste de Montenegro tenha sido negativo e a ocorrência de infeç̧ões concomitantes (como hepes simples, pneumonia lobar, amebíase) não fossem mais graves que o habitual. Estes achados confirmam a imunodeficiência dos portadores de LCD, específica à infecção leishmaniótica, confinada à imunidade celular $^{4}{ }^{6}$. Esta deficiência pode ser congênita ou resultar da depressão primária da imunidade celular provocada pela infecção leshmaniótica ou algum evento ainda desconhecido ${ }^{8132632}$.

Já Akuffo e cols ${ }^{12}$ acreditam que as diferentes respostas nas duas formas de leishmaniose (cutânea e cutânea difusa) são devidas à variação nas propriedades antigênicas dos agentes infectantes. Observaram que os linfócitos de pacientes com LCD e de controles normais produzem mais interleucinas 2 (IL-2) e interferon-gamma (-IFN), quando estimulados com antígenos de promastigotas provenientes de pacientes com leishimaniose cutânea. Haveria nos parasitos causadores da LCD, antígenos especificamente supressores, os quais inibiriam a produção das linfocinas.

O acompanhamento por tempo prolongado dos pacientes atesta o caráter evolutivo crônico da doença, observando-se que a infecção inicial ocorreu em $84 \%$ dos casos no início da $1^{\text {a }}$ década de vida, confirmando o achado de outros autores ${ }^{101421}$. Não foi observado o caráter familiar, como citam Diaz e cols ${ }^{16}$ na República Dominicana, pois durante a 
Costa JML, Saldanha ACR, Silva ACM, Serra Neto A, Galvão CES, Silva CMP, Silva AR. Estado atual da leishinaniose cutânea difusa (LCD) no Estado do Maranhão. II. Aspectos epidemiológicos, clínico-evolutivos. Revista da Sociedade Brasileira de Medicina Tropical 25:115-123, abr-jun, 1992.

Tabela 3 - Distribuição quanto ao tempo de doença dos seis pacientes estudados correlacionando com o tratamento e evolução.

\begin{tabular}{cccccc}
\hline Pacientes & $\begin{array}{c}\text { Início da } \\
\text { doença }\end{array}$ & $\begin{array}{c}\text { Diagnóstico } \\
\text { (ano) }\end{array}$ & $\begin{array}{c}\text { Tratamentos } \\
\text { anteriores }\end{array}$ & $\begin{array}{c}\text { Tratamento } \\
\text { atual }\end{array}$ & Evolução \\
& & and
\end{tabular}

\begin{tabular}{|c|c|c|c|c|c|}
\hline MECS* & 1974 & 1978 & $\mathrm{~T} 1 \mathrm{~T} 4 \mathrm{~T} 5 \mathrm{~T} 6$ & $\mathrm{~T} 7$ & clinicamente melhorado \\
\hline RNMG & 1980 & 1983 & $\mathrm{~T} 1$ & T1 T3 & clinicamente melhorado \\
\hline AAA & 1978 & 1983 & $\mathrm{~T} 1 \mathrm{~T} 2$ & T1 T3 & $\begin{array}{l}\text { clinicamente intercalando } \\
\text { períodos de melhora e } \\
\text { exacerbação }\end{array}$ \\
\hline RNPS & 1983 & 1989 & $\mathrm{~T} 1 \mathrm{~T} 2$ & $\mathrm{~T} 1 \mathrm{~T} 2$ & $\begin{array}{l}\text { quadro clínico involuindo } \\
\text { lentamente }\end{array}$ \\
\hline MCMA & 1987 & 1990 & $\mathrm{~T} 1$ & T1 T3 & $\begin{array}{l}\text { quadro clínico involuindo } \\
\text { lentamente }\end{array}$ \\
\hline ROF & 1976 & 1977 & $\mathrm{~T} 1 \mathrm{~T} 2 \mathrm{~T} 3$ & $\mathrm{~T} 1 \mathrm{~T} 3$ & $\begin{array}{l}\text { lesões nódulo tumorais } \\
\text { exulcerada totalmente } \\
\text { involuídas, presistindo } \\
\text { pequenos nódulos infil- } \\
\text { trados a nível da face }\end{array}$ \\
\hline
\end{tabular}

\begin{tabular}{llll}
\hline Legenda: Tratamento & T1 - glucantime & T4 - rifampicina & T7 - pentamidina \\
& T2 - anfotericina B & T5 - metronidazol & \\
& T3 - interferon gama & T6 - sulfona &
\end{tabular}

* Paciente encontra-se em Belém-PA, fazendo uso de pentamidina (Instituto Evandro Chagas).

realização do nosso trabalho, tivemos a oportunidade de acompanhaar a irmã do paciente (R.N.M.G.), que contraiu leishmaniose cutânea na mesma localidade do irmão, desenvolvendo a forma verrucóide com intradermorreação de Montenegro positiva e resposta ao antimoniato- $\mathrm{N}$-metilglucamina (glucantime), na dose de $20 \mathrm{mg} / \mathrm{kg} /$ peso/dia durante 40 dias.

Quando à refrateriedade ao uso do antimoniato$\mathrm{N}$-metil-glucamina (glucantime), observa-se uma resposta inicial satisfatória, porém temporária, com posterior reativação das lesões no mesmo local ou em locais diferentes. Bryceson ${ }^{8}$, usando 11 drogas no tratamento da LCD na Etiópia, observou que a pentamidina e $a$ anfotericina $B$ foram as drogas às quais o parasito foi constantemente sensivel. Neste período, geralmente, os pacientes apresentam padrão histológico tuberculóide, com reação de Montenegro positiva (imunoconversão) ${ }^{3} 62223$. Segundo esse autor, o emprego de qualquer droga efetiva nesta fase possibilitaria a "cura da doença".

\section{SUMMARY}

The authors describe a retrospective and prospective study of 6 patients with diffuse cutaneous leishmaniasis observed in the State of Maranhão, since 1974. The patients come from different rural regions of the state and in all of them Leishmania (Leishmania) amazonensis was the cause five of the patients initiated their disease in the first decade of life. All the patients first had a solitary, nodular lesion, that after a variable period of time, 
Costa JML, Saldanha ACR, Silva ACM, Serra Neto A, Galvão CES, Silva CMP, Silva AR. Estado atual da leishmaniose cutânea difusa (LCD) no Estado do Maranhão. II. Aspecios epidemiológicos, clínico-evolutivos. Revista.da Sociedade Brasileira de Medicina Tropical 25:115-123, abr-jun, 1992.

disseminated and acquired other aspects. Sequentially the patients presented multiple nodular and ulcerative lesions, negative leishmania skin-tests and a refractory response to the therapeutic schedules used up to the present.

Key-words: Diffuse cutaneous leishmaniasis. Leishmania (Leishmania) amazonensis. State of Maranhão.

\section{REFERÊNCIAS BIBLIOGRÁFICAS}

1. Akuffo HO, Fehniger TE, Britton S. Differential recongnition of leishmania aethiopica antigens by lymphocytes from patients with local and diffuse cutaneous leishmaniasis. Journal of Immunology 141:2461-2466, 1988.

2. Akuffo HO, Shurr E, Anderson G, Yamanekerhan $T$, Britton S. Responsiveness in diffuse versus local cutaneous leishmaniasis is due to parasite differences. Scandinavian Journal of Immunology 26: 717-721, 1987.

3. Barrientos PL. Um caso típico de leishmaniose cutâneo-mucosa. Memórias do Instituto Oswaldo Cruz 46-415-418, 1948.

4. Bittencourt AL, Freitas LAR. Leishmaniose tegumentar difusa - Aspectos anátomo-patológicos. Medicina cutânea I. L.A. 11:256-270, 1983.

5. Bittencourt AL, Guimarães NA. Imunopatologia da leishmaniose tegumentar difusa. Medicina Cutânea I. L.A. 4:395-402, 1968.

6. Bryceson ADM. Diffuse cutaneous leishmaniasis in Ethiopia, III Immunological studies. Transactions of the Royal Society of Tropical Medicine and Hygiene 64:380-387, 1970.

7. Bryceson ADM. Diffuse cutaneous leshmaniasis in Ethiopia, II. Treatment. Transactions of the Royal Society of Tropical Medicine and Hygiene 64:369379, 1970.

8. Carvalho EM, Badaró R. Absence of Gamma interferon and interleukin 2. Production curing Active Visceral Leishmaniasis. Journal of Clinical Investigation 76: 2066-2069, 1985.

9. Convit J, Alarcon CJ, Medina R, Reyes O, KerdelVegas F. Leishmaniasis tegumentaria difusa. Nueva entidad clinico-patologica $Y$ parasitaria. Archives Venezuelanos Patologia Tropical e Parasitologia Medica 3: 219-251, 1959.

10. Convit J, Castellanos PF, Ulrich M, Costés $M$, Rondon A, Pinardi ME, Rodrigues N, Bloom BR, Formica S, Valecillos L, Bretana A. Immunotherapy of localized, intermediate and diffuse forms of american cutaneous leishmaniasis. The Journal of Infectious Diseases 160: 104-115, 1989.

11. Convit J, Kerdel-Vegas F. Disseminated cutaneous leishmaniasis. Archives of Dermatology 91: 439$447,1965$.
12. ConvitJ, Lapenta P. Sobre um caso de leishmaniasis disseminada. Revista Patologia Clínica (Caracas) 17: 153-158, 1946.

13. Convit J, Pinardi ME, Rondon AJ. Diffuse cutaneous leishmaniasis: a disease due to an immunological defect on the host. Transactions of the Royal Society of Tropical Medicine and Hygiene 66: 603-610, 1972.

14. Convit J, Reyes O, Kerdel F. Disseminatede anergic american leishmaniasis. A.M.A. Archives of Dermatology 76: 213-217, 1957.

15. Costa JML, Saldanha ACR, Silva CMP, Serra-Neto A, Galvão CES, Godinho AMR, Silva AC, Mendes WS, Silva ACM. Estágio atual da leishmaniose cutânea difusa (LCD) no Estado do Maranhão. I. Relato Preliminar. Revista da Sociedade Brasileira de Medicina Tropical 24: 59-60, 1991.

16. Diaz HB, Martinez D, Quinones M, Estevez FN. Leishmaniose enérgica na República Dominicana Estudo de 20 casos. Anais Brasileiros de Dermatologia 60 (supl1):229-236, 1985.

17. Kadivar DMH, Soulsay EJL. Model of disseminated cutancous leishmaniasis. Science 190:1198-1220, 1975.

18. Lainson R, Shaw JJ. Las leishmanias y la leishmaniasis del Nuevo Mundo, con particular referencia al Brasil. Bulletin of Sanitary Panamerican 76: 93-119, 1974.

19. Mayer M, Convit J, Pifano F. Estudios experimentales con una cepa de leishmania braziliensis proveniente de un caso de leishmaniasis tegumentaria diseminada aspecto lepromatoso. Archives Venezoelanos Medicina Tropical 1:183192, 1949.

20. Medina R, Romero J. Estudio sobre la leishmaniasis tegumentaria en Venezuela. Dermatologia Venezuelana 1:33-86, 1957.

21. Medina R, Romero J. Estudio clinico e parasitológico de una nueva cepa de Leishmania. Archives Venezoelanos Patologia Tropical Y Parasitologia Médica 3:298-326, 1959.

22. Medina R, Romero J. Leishmania pifanoi nsp. El agente causal de la leishmaniasis tegumentaria difusa. Archives Venezoelanos Medicina Tropical e Parasitologia Médica 4:349-353, 1962.

23. Orsini O. Leishmaniose em Minas Gerais. Brasil 
Costa JML, Saldanha ACR, Silva ACM, Serra Neto A, Galväo CES, Silva CMP, Silva AR. Estado atual da leishmaniose cutânea difusa (LCD) no Estado do Maranhão. II. Aspectos epidemiológicos, clínico-evolutivos. Revista da Sociedade Brasileira de Medicina Tropical 25:115-123, abr-jun, 1992.

Médico 76:766, 1940.

24. Pereira LIA. Leishmaniose Tegumentar - Estudo terapêutico baseado nos aspectos clínicos e imunológicos. Revista de Patologia Tropical 8:191391, 1974.

25. Petersen EA, Neva FA, Barral A, Correa-Coronas R, Diaz-BogaertH, Martinez D, Ward FE. Monocyte supression of antigen-specific limphocyte responses in diffuse cutaneous leismaniasis patients from the Dominican Republic. The Journal of Immunology 132:2603-2606, 1984.

26. Petersen EA, Neva FA, Oster CN, Diaz-Bogaert H. Specific inhibition of lymphocyte proliferation responses by adherent supression cells in diffuse cutaneous leishmaniasis. The New England Journal of Medicine 306:387-391, 1982.

27. Porto Marques A, Portugal H. Leishmaniose tegumentar difusa. O Hospital 57:11-23, 1960.

28. Rabello FE. Leishmaniose cútis difusa. Anais Brasileiros de Dermatologia 53:157, 1978.
29. Silva AR, Martins G, Melo JEM, Araújo JR, Mendes MG. Surto epidêmico de leishmaniose tegumentar americana ocorrido na colonização agrícola de Buriticupu (Estado do Maranhão) Brasil. Revista do Instituto de Medicina Tropical de São Paulo 21:43-50, 1979.

30. Silva AR, Mendes JR, Rodrigues MLM, Carvalho ZS, Reis FMP, Melo JEP, Morais JCO. Leishmaniose cutânea difusa (LDC). Registro de um caso em Buriticupu (Estado do Maranhão). Revista do Instituto de Medicina Tropical de São Paulo 23:1-4, 1981.

31. Silva D. Leishmaniose anérgica hansenóide. Anais Brasileiros de Dermatologia 53:163-168, 1978.

32. Silva F. Forma raríssima de Leishmaniose Tegumentar. Leishmaniose dérmica não ulcerada em nódulos e extensas placas infiltradas e hiperpigmentadas. Separata do Volume de Reunião Anual da Dermato-Sifilógrafos Brasileiros, Rio de Janeiro, 97-103p, 1945. 